\title{
Compensatory Rapid Switching of Binasal Inputs in the Olfactory Cortex
}

\author{
Shu Kikuta, ${ }^{1,2}$ Hideki Kashiwadani, ${ }^{1}$ and Kensaku Mori ${ }^{1}$ \\ Departments of ${ }^{1}$ Physiology and ${ }^{2}$ Otolaryngology, Graduate School of Medicine, The University of Tokyo, Tokyo 113-0033, Japan
}

Odors are inhaled through the nostrils into two segregated nasal passages and detected by sensory neurons in the bilateral olfactory epithelia. Airflow through the two nasal passages is usually asymmetrical because of alternating changes in nasal mucosal congestion. Here we show that neurons in the anterior olfactory nucleus (AON) of the adult rat olfactory cortex are ordinarily dominated by ipsi-nasal inputs and that binasal neurons in the AON respond to ipsilateral and contralateral nasal inputs with nearly equivalent odorant category selectivity. Deprivation of ipsilateral nasal inputs by unilateral nostril obstruction greatly enhanced the response to contralateral odor stimulation, in a reversible manner, in $\sim 33 \%$ of AON neurons within only several minutes. In $27 \%$ of AON neurons that showed spike responses induced by the inspiration of room air, ipsilateral nasal obstruction initially suppressed respiration phase-locked spike discharges and, several minutes later, induced respiration phase-locked discharges with longer delays between inspiration and response. Recordings from AON neurons in rats with anterior commissure (AC) transection indicated that the resumed respiration phase-locked discharges with longer delays were mediated by the contralateral pathway via the AC. The ipsi-nasal occlusion-induced switching of nasal inputs to individual AON neurons shows that a subset of AON neurons in the adult rat has neuronal mechanisms for rapid nostril dominance plasticity, which may enable both right and left olfactory cortices to preserve their responsiveness to the external odor world, despite reciprocal changes in nasal airflow.

Key words: olfactory cortex; anterior olfactory nucleus; nostril obstruction; binasal inputs; compensation; homeostatic plasticity

\section{Introduction}

Airflow through the two, right and left nasal passages is usually asymmetrical. A slight to mild inflammatory rhinitis causes one of the typical symptoms, a spontaneous alternative unilateral obstruction because of the nasal congestion. Complete or partial obstruction of a unilateral airway occurs even in normal physiological conditions, and has been reported as nasal cycle (BojsenMoller and Fahrenkrug, 1971; Eccles, 2000a). Such a collapse of a unilateral nasal airway prevents environmental odor information from reaching not only the olfactory epithelium (OE) but also the olfactory bulb $(\mathrm{OB})$ on the blocked side; this occurs because the olfactory sensory neurons in the OE selectively project their axons to the ipsilateral (ipsi)-OB (Fig. 1) (Mori et al., 1999). The mitral and tufted cells in the $\mathrm{OB}$ also selectively project their axons to the ipsi-olfactory cortex (OC) (Scott et al., 1980; Neville and Haberly, 2004). Based on these activities, the question is raised, whether the olfactory cortex in the odor-deprived side is completely isolated from the external odor world.

In the developing visual cortex, monocular deprivation leads

\footnotetext{
Received June 27, 2008; revised Aug. 18, 2008; accepted 0ct. 4, 2008.

This work was supported by grants-in-aid for scientific research on priority areas from Ministry of Education, Culture, Sports, Science, and Technology (K.M.) and from Japan Society for the Promotion of Science (K.M., H.K.). We thank Drs. T. Yamasoba, K. Kondo (Department of Otolaryngology), M. Yamaguchi, and H. Nagao for critically reading this manuscript; and members of the Departments of Otolaryngology and Physiology for useful discussion. The authors declare no competing interests.

Correspondence should be addressed to Kensaku Mori, Department of Physiology, Graduate School of Medicine, The University of Tokyo, 7-3-1 Hongo, Bunkyo-ku, Tokyo 113-0033, Japan. E-mail: moriken@m.u-tokyo.ac.jp. D01:10.1523/JNEUROSCI.3106-08.2008

Copyright $\odot 2008$ Society for Neuroscience ～0270-6474/08/2811989-09\$15.00/0
}

to a functional weakening of inputs from the deprived eye and a persistent enhancement of inputs from the non-deprived eye (Hensch, 2005). The adult mouse visual cortex also shows a form of ocular dominance plasticity; depriving the dominant contralateral eye of vision leads to a persistent enhancement of the weaker ipsilateral eye inputs (Sawtell et al., 2003). Because of the frequent occurrence of unilateral nasal occlusion in the olfactory system, we hypothesized that when ipsi-nasal inputs are deprived by nasal occlusion, neurons in the adult rodent OC may enhance the inputs from the contralateral, nonoccluded nose.

The anterior olfactory nucleus ( $\mathrm{AON})$ is found at the most rostral region of the OC. AON neurons project their axons as association fibers to the ipsi-OC, and to the contralateral (contra)-AON and contra-OC via the anterior commissure (AC) (Fig. 1) (Schoenfeld and Macrides, 1984; Brunjes et al., 2005). It appears that the $\mathrm{AON}$ receives axonal inputs via the $\mathrm{AC}$ from the contra-AON or contra-OC (Haberly and Price, 1978), and thus receives sensory signals from both the ipsi- and contra-OEs (Wilson, 1997; Lei et al., 2006). On ipsi-nasal obstruction, the AON may obtain external odor information from the pathway consisting of the contra-OE, contra-OB, contra-AON/OC, and AC (Fig. 1 , blue pathway).

In the present study, we addressed the question whether neurons in the AON receive similar olfactory information from the ipsi-OE and the contra-OE. For selective unilateral nasal stimulation with odorants, we positioned a thermoplastic external nasal septum to prevent the odors delivered in front of one nostril from spreading into the contralateral nostril (Figs. 1, 2A). In 


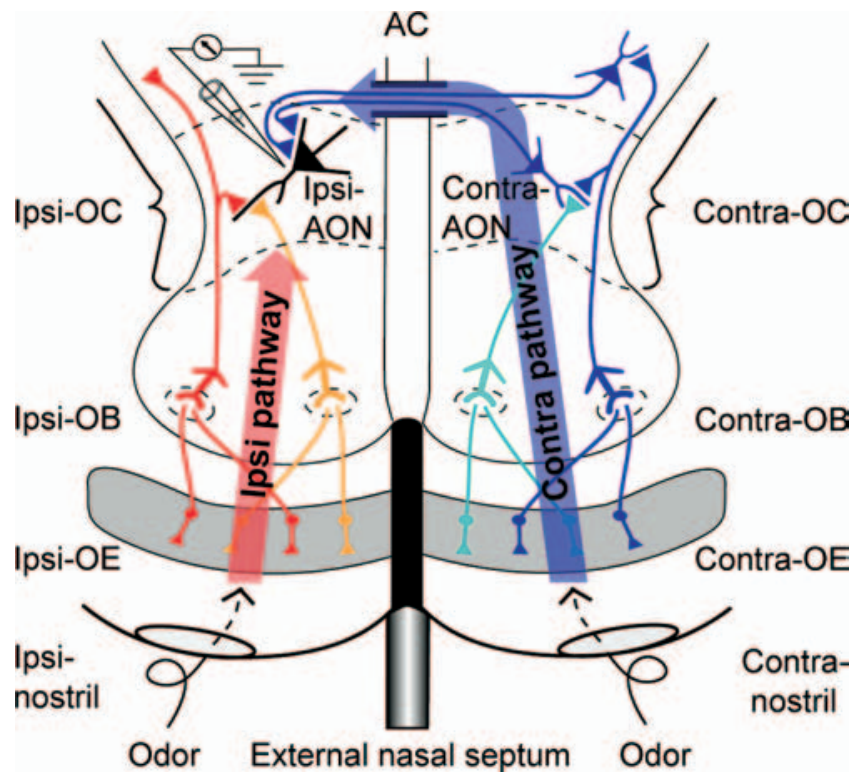

Figure 1. Neuronal pathways from the ipsilateral olfactory epithelium (Ipsi-pathway) and contralateral olfactory epithelium (contra-pathway) to the anterior olfactory nucleus (AON). Single-unit recordings (shown by a micropipette) were obtained from AON neurons in the animal's right olfactory cortex (OC). Shown are schematic depictions of the ipsi-nasal pathway $($ Ipsi- $0 \mathrm{E} \rightarrow|\mathrm{psi}-\mathrm{OB} \rightarrow| \mathrm{psi}-\mathrm{AON})$ (red), and the contra-nasal pathway (contra- $\mathrm{OE} \rightarrow$ Contra$\mathrm{OB} \rightarrow$ Contra-AON/OC $\rightarrow$ Ipsi-AON) (blue) to the AON. Connection between ipsi- and contraAONs is either monosynaptic (as shown here) or polysynaptic (not shown). AC, anterior commissure; $0 \mathrm{E}$, olfactory epithelium.

addition, a chitin membrane was pasted onto one of the nostrils. We recorded the single-unit spike responses of AON neurons to stimulation of ipsilateral or contralateral OEs with distinct odorant categories, in the brain of urethane-anesthetized rats. We then examined the effect of unilateral nasal obstruction on the odor response to contra-nasal stimulation.

\section{Materials and Methods}

Animals. All experiments were performed in accordance with the guidelines of the Physiological Society of Japan and were approved by the experimental animal research committee of the University of Tokyo. Wistar rats (male, 280-350 g; Japan SLC) were anesthetized with urethane $(1.2 \mathrm{~g} / \mathrm{kg})$ (Nagayama et al., 2004) and placed in a stereotaxic apparatus (SR-6R; Narishige). Body temperature was maintained at $37.5^{\circ} \mathrm{C}$ by a homeothermic heat pad system (MK-900; Muromachi Kikai). Respiration was monitored by measuring chest movement with a strain gauge (TR-651T; Nihon Kohden), or by examining nasal airflow with a thermistor (BAT-12, Physitemp Instruments). The thermistor probe was placed in the nasal vestibule contralateral to the recording side, without touching the nasal mucosa. We subjected seven rats to AC transection using a miniature edge blade (501251; World Precision Instruments). The success of the AC transection in each rat was confirmed histologically after the experiment was completed.

Odor stimulation. For uni-nasal odor stimulation, thermoplastic material was used to construct an external nasal septum which fit the external shape of the rat nose. In addition, one nostril was obstructed by pasting on a chitin membrane $(0.5 \mathrm{~cm}$ in diameter and moisturized with saline), which allowed noninvasive obstruction or re-opening of one nostril (Fig. 2C). To stimulate the $\mathrm{OE}$ with each odorant category, we first used the method of placing test tubes containing diluted odorants (5\% in odorless mineral oil) in front of a nostril at a distance of $2 \mathrm{~cm}$. After monitoring the odorant category selectivity of individual AON neurons, we then examined the effect of nostril obstruction using a custom made air-dilution olfactometer fitted with Teflon tubing, controlled by a program written in Labview software (National Instruments). Odorant vapor was produced by flowing clean air through disposable glass microfi- ber syringe filters $(2.7 \mu \mathrm{m}$ pore size; Whatman) loaded with $30 \mu \mathrm{l}$ of liquid odorant. Each odorant stream $(300 \mathrm{ml} / \mathrm{min})$ was mixed with a clean air stream $(200 \mathrm{ml} / \mathrm{min})$ to produce a total flow rate of $500 \mathrm{ml} / \mathrm{min}$ in a Teflon tube. The outlet of this tube was placed $2 \mathrm{~cm}$ in front of the ipsi-nostril (recorded side) or the contra-nostril. An exhaust pipe was placed over the head of the rat to suck up any stray odorants.

Odorants. Ten odorant categories (sulfide, ester, terpene hydrocarbon, acid, ether, ketone, aldehyde, alcohol, lactone and phenol) were used (supplemental Fig. 1, available at www.jneurosci.org as supplemental material). A mixture of five representative odorants was used for stimulation with an individual category. In some experiments, stimulation with individual component odorants was also performed. The odorants were a kind gift from T. Hasegawa (Tokyo, Japan), or were purchased from Sigma-Aldrich, Tokyo Kasei Organic Chemicals, or Nacalai tesque.

Electrophysiology. For extracellular single-unit recordings, a small hole was made in the skull above the AON (3.8-4.7 $\mathrm{mm}$ anterior to the bregma, $1.7-2.3 \mathrm{~mm}$ lateral from the midline). A glass micropipette (10-20 M $\Omega$ ) filled with 2\% Chicago Sky Blue 6B (Tocris Bioscience) in $0.5 \mathrm{M}$ sodium acetate was inserted vertically into the pars principalis of the AON (Brunjes et al., 2005).

After single-unit recordings, a negative current was applied so that the recorded sites could be marked by the dye ( $20 \mu \mathrm{A}, 2 \mathrm{~min})$. The dyemarked sites were examined histologically. The signals of single-unit activities were amplified (AB-610J, Nihon Kohden), filtered (150-10 $\mathrm{kHz}$ ) (EW-610J, Nihon Kohden), and stored on a computer.

For extracellular single-unit recordings from $\mathrm{OB}$ neurons (two rats), the bone above the OB and lateral olfactory tract (LOT) was removed by using a dental drill. A concentric, bipolar stainless electrode (EKC 5002K; Eikoh Kagaku) was inserted into the LOT (3.0 $\mathrm{mm}$ anterior from the bregma, $3.5 \mathrm{~mm}$ lateral from the midline, $\sim 6 \mathrm{~mm}$ from the brain surface) for electrical stimulation (once every $3 \mathrm{~s}, 100 \mu \mathrm{s}$ in duration). A glass micropipette (1-5 M $\Omega$ ) filled with $4 \mathrm{M} \mathrm{NaCl}$ was inserted into the OB. Single-unit activities were recorded exclusively from the external plexiform layer or mitral cell layer.

For multisite single-unit recordings from AON neurons, a siliconbased stereotrode (16 or 32 channels; Neuro Nexus Technologies) was used. Recording sites in the probe were spaced at $100 \mu \mathrm{m}$ intervals. After removing the skull and dura matter, the stereotrode was inserted into the AON through the frontal cortex. Monitoring of the LOT-evoked local field potentials indicated that most of the recording sites were placed at layer II of the AON, pars principalis. Signals were amplified (AB-610 J; Nihon Koden), filtered (150 Hz-10 kHz) (EW-610J; Nihon Koden), digitized (Power 1401; Cambridge Electronic Design) and stored on a computer. In recordings made with the stereotrode, single units were isolated from recorded signals in each recording site by using template matching and principal component analysis methods. Raster plots and peristimulus time histograms were obtained as described previously (Nagayama et al., 2004). In the raster plots, trials are shown in chronological order from bottom to top. After the recording session, small lesions were made by current injections ( $5 \mu \mathrm{A}$ during $5 \mathrm{~s}$ ) for later histological examination of the recorded sites.

For electro-encephalogram (EEG) recording, a stainless-steel screw was threaded into the bone above the occipital cortex. Another screw was threaded into the bone above the cerebellum, and used as a reference.

Analysis. Spike2 software (Cambridge Electronic Design) was used off-line to analyze spike firing rate, and to make raster representations, peristimulus time histograms $[1 \mathrm{bin}=0.1 \mathrm{~s}$ (Figs. 2,3$) ; 1 \mathrm{bin}=0.3 \mathrm{~s}$ (see Fig. 6; supplemental Fig. 1, available at www.jneurosci.org as supplemental material)] and respiratory phase histograms (one respiration $=24$ bins) of spikes. The response magnitude (R.M.) to odor stimulation (see Figs. 6,7 ) was calculated by subtracting the number of spikes during the $3 \mathrm{~s}$ period immediately before odor stimulation from the number of spikes observed during the $3 \mathrm{~s}$ period of odor stimulation, and was expressed as firing rate (number of spikes/s).

In rats under urethane anesthesia, the neocortical EEG alternates between the slow-wave state and the fast-wave state (Murakami et al., 2005). To minimize the influence of state-dependent sensory gating, all spike responses to odorants were obtained during the fast-wave state.

Based on responses to odor stimulation, we classified AON neurons 


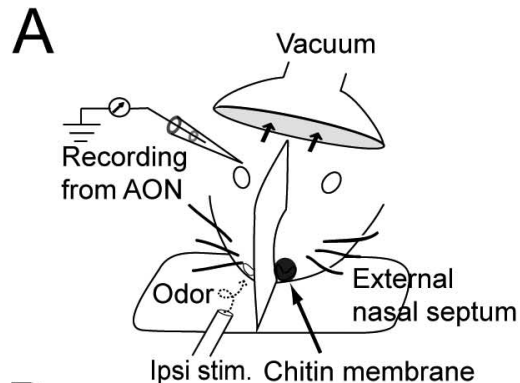

C

B

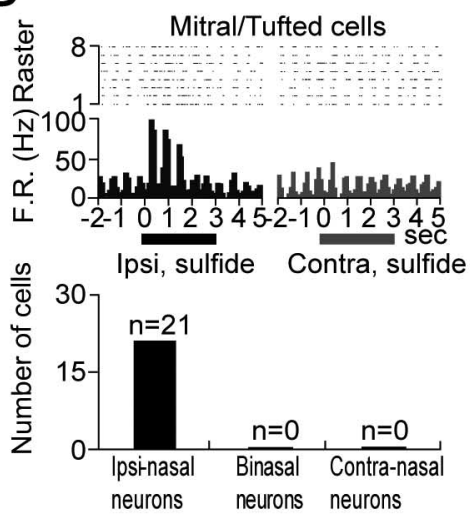

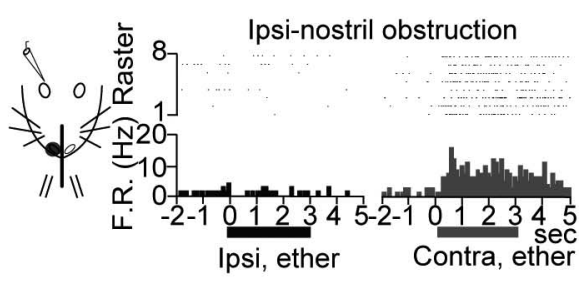
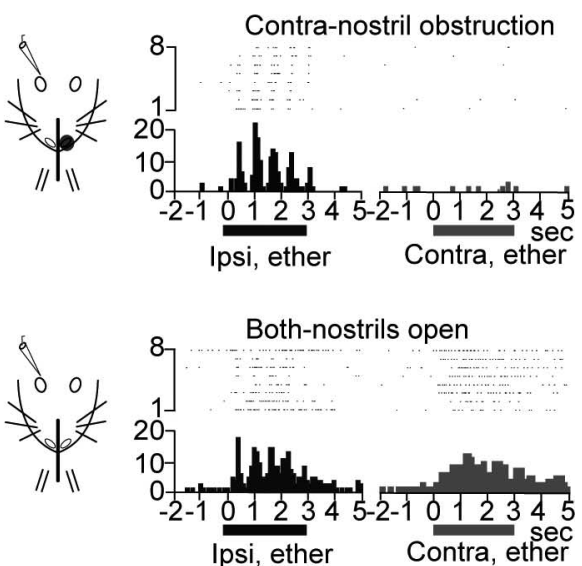

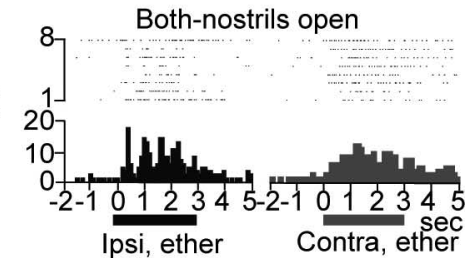

Figure 2. Experimental procedure used for selective unilateral $O E$ stimulation. $A$, Schematic depiction of the unilateral $O E$ stimulation method. An external nasal septum made of thermoplastic material was used to separate the space in front of the right nostril from that of the left nostril. In some experiments, a chitin membrane (black disk) was also pasted onto one of the nostrils. $\boldsymbol{B}$, Mitral/tufted cells in the $O B$ showed an excitatory response to ipsi-OE stimulation only. Raster representation and peristimulus time histograms of the spike response of a mitral/tufted cell to ipsi-OE (upper left, red) and contra-OE (upper right, blue) stimulation are shown. F.R., firing rate. The bottom histogram shows the number of ipsi-nasal neurons, binasal E-E (ipsi-excitation, contra-excitation) type neurons and contra-nasal neurons that were detected in the OB. C, Excitatory spike responses of an AON neuron to ipsi-nasal (left column) and contra-nasal (right column) odor stimulation in a rat whose nostrils were subjected to unilateral nostril obstruction with a chitin membrane (upper and middle), or were both open. Ipsi-nostril obstruction selectively abolished responsiveness to ipsi-0E stimulation (upper left), whereas contra-nostril obstruction selectively abolished responsiveness to contra-0E stimulation (middle right), while leaving intact the response to 0 E stimulation in the nonoccluded side.

A
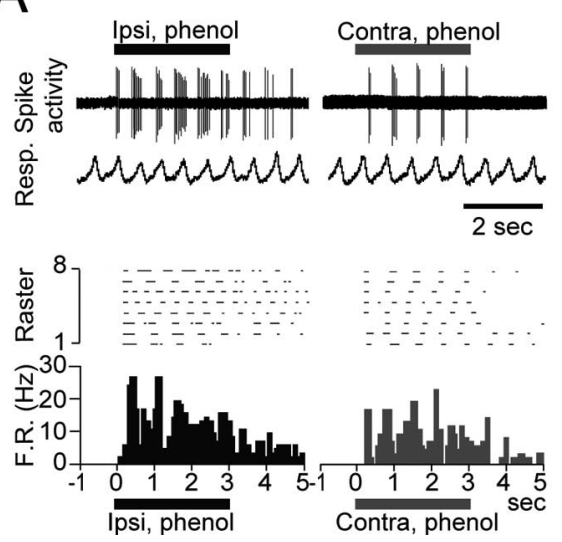

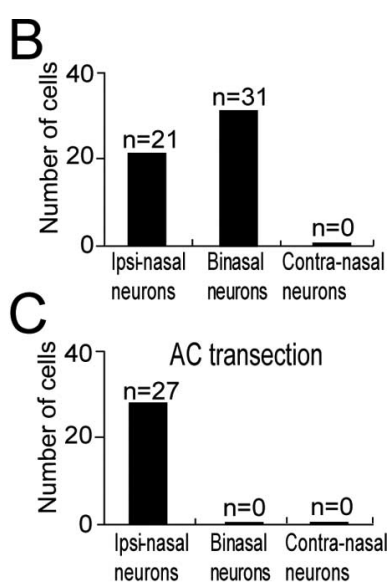

Figure 3. AON neurons receive contra-nasal inputs via anterior commissure. $A$, Spike responses of a binasal (E-E type) AON neuron to ipsi-nasal (upper left, red bar) and contra-nasal (upper right, blue bar) odor stimulation. Shown are a raster representation of the spike responses (middle) and peristimulus time histograms (bottom). Colored bar, duration of odor stimulation (phenol category, $3 \mathrm{~s}$ ). The ascending and descending phases of the trace in the respiration monitor (Resp.) indicate inspiration and expiration, respectively. $\boldsymbol{B}, \boldsymbol{C}$, The numbers of ipsi-nasal, binasal (E-E type), and contra-nasal neurons in the AONs of $(\boldsymbol{B})$ control rats (with intact $A C)$ and $(C) A C$-transected rats.

into two types: binasal neurons and ipsi-nasal neurons. Binasal neurons showed excitatory spike responses to both ipsi-OE and contra-OE stimulation with at least one odorant category. Thus, we designated these neurons binasal excitation-excitation (E-E) type neurons. Most of the ipsi-nasal neurons showed excitatory spike response to ipsi-OE stimulation, but did not respond to contra-OE stimulation. Some of the ipsi-nasal neurons showed an excitatory spike response to ipsi-OE stimulation and a suppressive response to contra-OE stimulation. These neurons were designated excitation-inhibition (E-I) type neurons.

To estimate the degree of similarity between odorant category selectivity of ipsi-nasal input and that of contra-nasal input, we counted for each neuron the number of odorant categories (among 10 categories examined) that induced matched responses between ipsi-OE stimulation and contra-OE stimulation.

For each odorant category, the bilateral match response was determined as +1 matched; excitatory ipsi-response plus excitatory contra-response, or no ipsi-response plus no contra-response or as 0 nonmatched; excitatory ipsi-response plus no contra-response, or no ipsi-response plus excitatory contraresponse). For each cell, the number of bilateral category matches was determined by summating all +1 matched responses. The averaged number of the bilateral category matches $(n=$ 13 cells) was then calculated (see Fig. 5C).

For 31 binasal neurons, the most effective odorant category for activating individual neurons was determined. In each of the binasal neurons, we compared the averaged magnitude of responses to ipsi-OE stimulation of the most effective odorant category with that of responses to contra-OE stimulation of the same category. The difference in the averaged magnitude of responses between ipsi-OE stimulation and contra-OE stimulation was statistically examined using paired $t$ test (significant level, $p<$ 0.05) (supplemental Fig. 4, available at www. jneurosci.org as supplemental material).

Stimulation of the OEs with each odorant category was performed at least three times for each OE (ipsi- or contra-OE). An excitatory spike response was defined as a significant increase (at least twofold) in the averaged firing rate during the $3 \mathrm{~s}$ period of odor stimulation, compared with that during the $3 \mathrm{~s}$ period immediately before odor stimulation. A suppressive spike response was defined as a significant decrease (one-half or less) in the averaged firing rate during the $3 \mathrm{~s}$ period of odor stimulation, compared with that during the $3 \mathrm{~s}$ period immediately before odor stimulation. Stimulation of the OEs with each odorant category was performed at least three times for each OE (ipsi- or contra-OE).

Histology. After electrophysiological experiments were completed, animals were deeply anesthetized with urethane and perfused with $0.9 \% \mathrm{NaCl}$ solution and then $4 \%$ paraformaldehyde (PFA) in phosphate buffer (PB, pH 7.4). The brains were postfixed in $4 \%$ PFA in $\mathrm{PB}$ at $4^{\circ} \mathrm{C}$ overnight. Coronal sections (50 $\mu \mathrm{m}$ thick) were cut on a microtome (VT1000 s; Leica), mounted onto glass slides, and stained with $\mathrm{cr}$ esyl violet. The dye-marked spots or small lesions made by current injections in the AON were visualized by using a microscope (MZ16FA; Leica). Locations of the recorded sites were reconstructed by referring to the dye mark or small lesions. 


\section{Results}

We used a stimulus panel of odors consisting of 10 odorant categories for nasal stimulation (supplemental Fig. 1, available at www.jneurosci.org as supplemental material). We first examined whether the external nasal septum was effective for selective odorant stimulation of the unilateral OE. We recorded extracellular, single unit activity from mitral/tufted cells in the $\mathrm{OB}$, which selectively project their axons to the ipsilateral OC. All of the recorded mitral/tufted cells showed excitatory responses to only ipsilateral nasal stimulation with odorants, and not to contralateral stimulation (Fig. 2B; supplemental Table 1, available at www.jneurosci.org as supplemental material). This indicated that contralateral nasal stimulation selectively stimulated the contralateral OE, or, alternatively, that the amount of odorants intruding from the ipsilateral nasal cavity was insufficient to drive the spike responses of mitral/tufted cells beyond the stimulation threshold.

We then recorded E-E (ipsi-excitation, contra-excitation) type AON neurons (Figs. $2 C, 3 A$ ) that showed excitatory responses both to ipsi-nasal and contra-nasal odor stimulation. We confirmed that obstruction of the ipsi-nostril by placement of a chitin membrane completely abolished the spike response of AON neurons to ipsi-nasal stimulation, but did not suppress their response to contra-nasal stimulation (Fig. 2C, top). Conversely, contra-nostril obstruction completely abolished spike responses to contra-nasal stimulation while leaving intact the response to ipsi-nasal stimulation (Fig. 2C, middle). These observations indicate the efficacy of the external nasal septum and chitin membrane for selectively stimulating only one OE.

\section{Responses of AON neurons to ipsi-nasal and contra-nasal odor stimulation}

We recorded $52 \mathrm{AON}$ neurons that showed excitatory response to at least one odorant category among the 10 categories tested (supplemental Fig. 1, available at www.jneurosci.org as supplemental material). Of these neurons, $\sim 60 \%$ (31 neurons) were excited by both ipsi-nasal and contra-nasal stimulation from at least one odorant category, and were thus identified as binasal E-E (ipsiexcitation, contra-excitation) type neurons (Fig. $3 A, B$, center column). Figure $3 A$ shows an example of excitatory responses of a binasal AON neuron to ipsi- and contra-nasal stimulation with phenol odorants. The remaining $40 \%$ of AON neurons were ipsinasal neurons that showed excitatory response only to ipsi-nasal stimulation (Fig. 3B, left column); three of these also showed suppressive responses to contra-nasal stimulation [E-I (ipsiexcitation, contra-suppression) type neurons; supplemental Figure 2, available at www.jneurosci.org as supplemental material]. The ipsi-nasal AON neurons received either no excitatory input from the contra-OE, or weak contra-nasal input below the threshold needed for a spike response. We found no AON neurons that showed an excitatory response to contra-nasal stimulation and no response to ipsi-nasal stimulation (contra-nasal neurons) (Fig. 3B).

The odor-induced spike responses of most AON neurons were phase-locked with the respiration cycle (Figs. 3A, 4). Responses to ipsi-nasal odor stimulation occurred largely during the inspiration phase or the initial part of the expiration phase (Fig. 4B), whereas contra-nasal odor responses generally occurred during the initial or later parts of expiration (Fig. $4 C$; supplemental Fig. $3 A$, available at www.jneurosci.org as supplemental material). Thus, the peak of the spike response to ipsinasal odor stimulation in the respiration phase histogram precedes that of the response to contra-nasal stimulation. This may

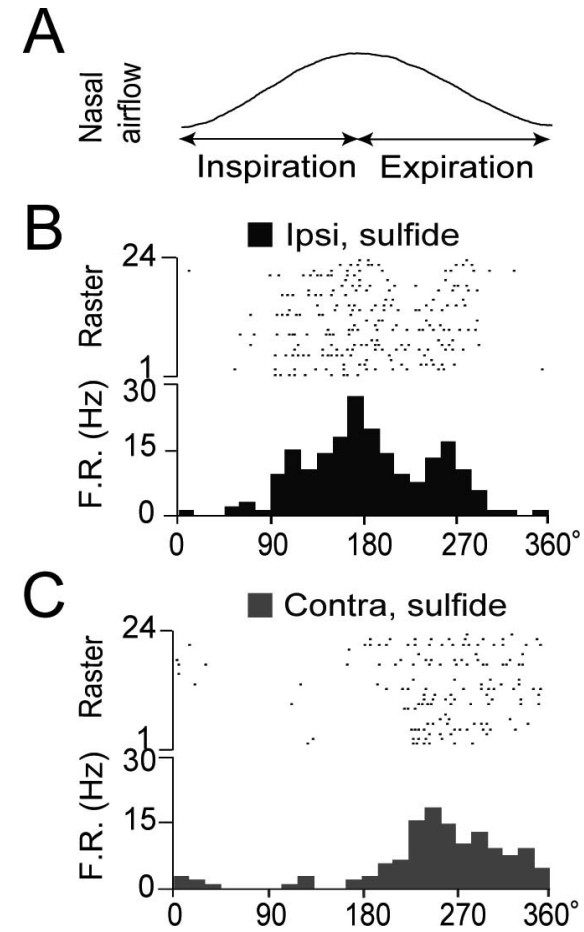

Figure 4. $\quad A-C$, Respiration phase histograms of spike responses of an $A O N$ neuron to ipsi-OE ( $\boldsymbol{B}$, red dots and bars) and contra-OE ( $\boldsymbol{C}$, blue dots and bars) odorant stimulation. In $\boldsymbol{A}$, a single respiration cycle is shown. The spike responses to ipsi- $0 \mathrm{E}$ and contra-0E stimulation peaked at the inspiration- expiration transition (red histogram) and the expiration phase (blue histogram), respectively. The response to ipsi-OE stimulation was greater than that to contra-OE stimulation.

occur because responses to contra-nasal stimulation are mediated by a longer pathway and a larger number of synaptic transmissions that include corticocortical synaptic interaction via the commissural fibers crossing the AC.

To confirm that the responses of binasal neurons to contranasal stimulation are mediated by the neuronal pathway traversing the AC, we surgically performed AC transection in four rats and examined the response to ipsi- or contralateral odor stimulation. The AON neurons in these rats indeed responded to ipsinasal stimulation but not to contra-nasal stimulation (Fig. 3C), indicating that $\mathrm{AC}$ mediates the sensory input from the contra-OE to the ipsi-AON.

Examination of odorant category selectivity of individual binasal neurons when odorants were introduced ipsi- or contranasally revealed that neurons were tuned to either a single odorant category or a specific combination of odorant categories (Fig. 5 ), as previously observed for neurons in the anterior piriform cortex (Yoshida and Mori, 2007). Surprisingly, in all 13 binasal E-E (ipsi-excitation, contra-excitation) type AON neurons examined in detail, the odorant category profile of ipsi-nasal inputs closely resembled that of the contra-nasal inputs (Fig. 5). However, for individual neurons, the contra-nasal-activating odorant categories were often a subset of the ipsi-nasal-activating odorant categories. In addition, in most cases the ipsi-OE stimulation response was larger than the contra-OE stimulation response for odorants within the same category (Fig. 3A; supplemental Fig. 4, supplemental Table 1, available at www.jneurosci.org as supplemental material) (one-way ANOVA; no significant differences among animals, paired $t$ test in 31 binasal neurons, $p<0.01$ ). Thus, individual binasal neurons receive nearly equivalent odor signals from right and left OEs in terms of odorant category pro- 


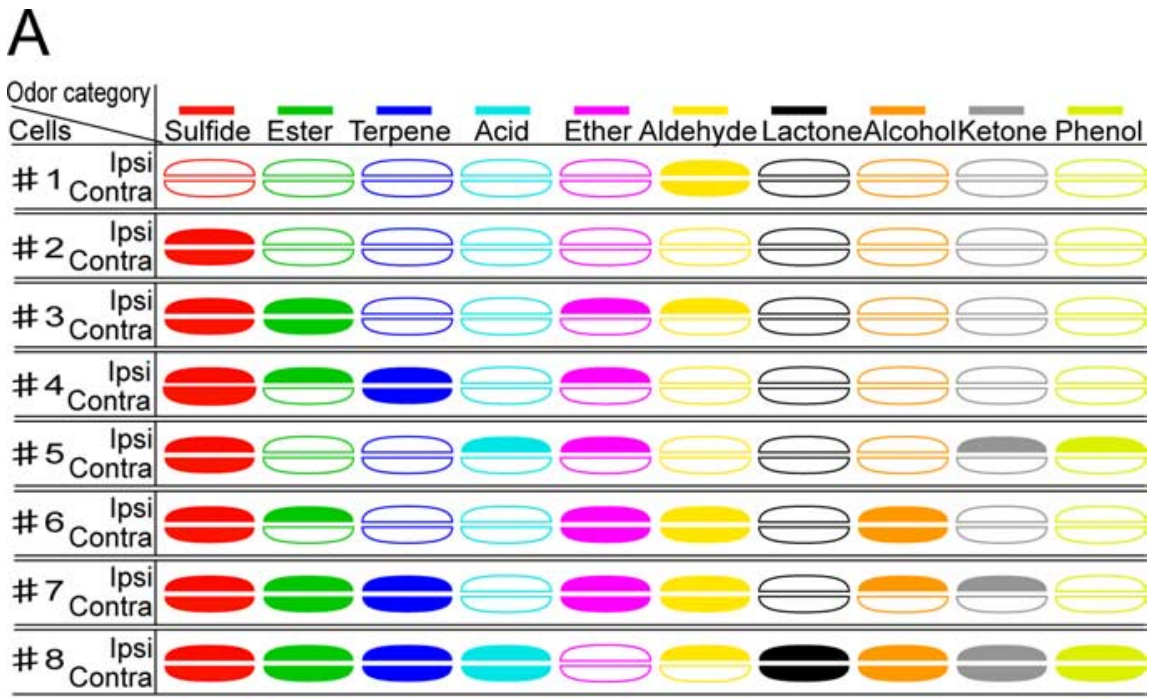

\section{B}

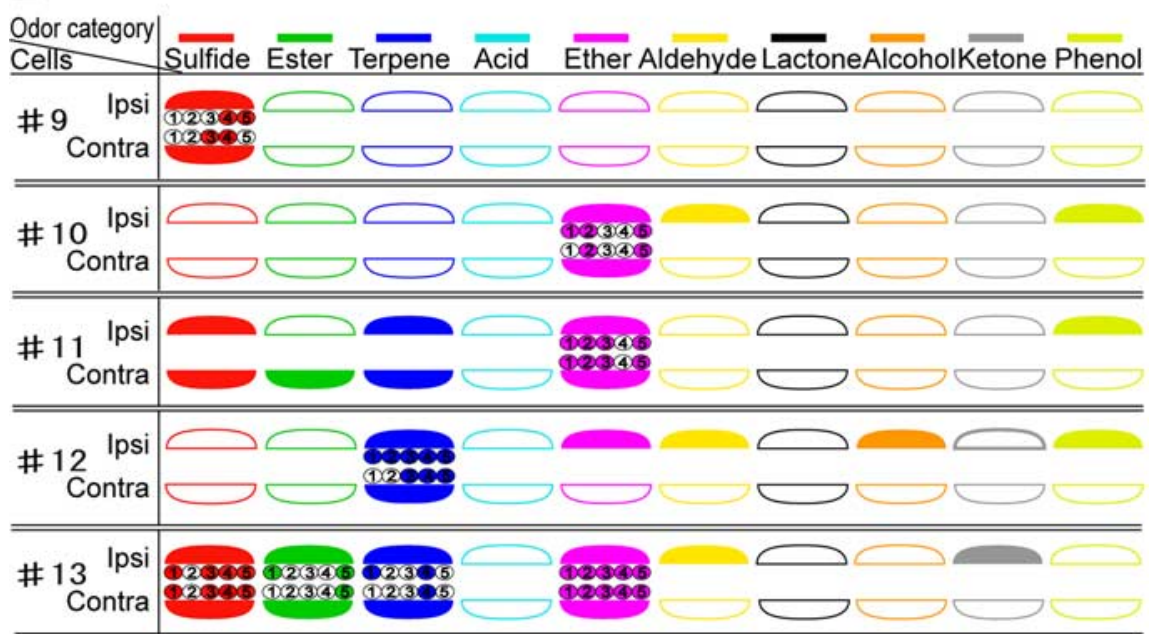

C

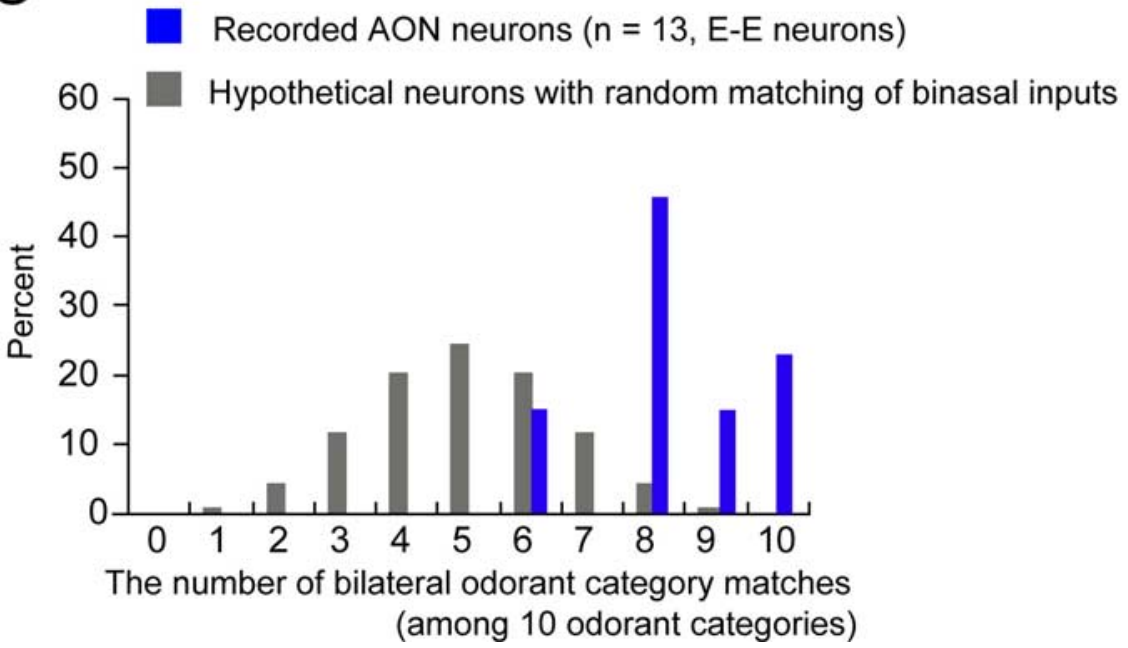

Figure 5. Individual AON neurons show nearly equivalent odorant category selectivity for ipsi- and contra-nasal inputs. $\boldsymbol{A}$, The odorant category selectivity of each neuron (1-8) is represented in each row. The filled and open marks indicate significant and absent spike responses to ipsi/contra- $\mathrm{OE}$ stimulation with individual odorant categories, respectively. The odorant category selectivity of AON neurons after ipsi- and contra-nasal inputs matched closely, but the latter categories were typically a subset of the former. $\boldsymbol{B}, 0$ dorant selectivity of individual $A O N$ neurons does not differ significantly, regardless of whether inputs are from the ipsi- or contra-OE. Small circles with numbers between the upper and lower marks indicate the response to an individual odorant file selectivity. In addition, the magnitude of ipsi-nasal inputs to the binasal neurons typically exceed those of contra-nasal inputs. This observation of binasal neurons together with the presence of ipsi-nasal AON neurons and the absence of contranasal neurons indicates that most $\mathrm{AON}$ neurons are dominated by ipsi-nasal inputs.

Deprivation of ipsi-nasal inputs rapidly enhances contra-nasal inputs to AON neurons

We next examined the effect of dominant ipsi-nasal input deprivation caused by ipsi-nostril obstruction on the response of individual AON neurons to contra-nasal stimulation. When both nostrils were left open, the cell pictured in Figure $6 \mathrm{~A}$ was observed to respond strongly to ipsi-OE stimulation with "ether" category odorants, but did not respond to contra-OE stimulation with the same odorants. When the ipsilateral nostril was blocked by placement of the chitin membrane at time 0 (Fig. 6A, filled triangle), the cell remained unresponsive to contra-OE stimulation for $\sim 200 \mathrm{~s}$, and then began responding strongly (Fig. 6Ac). On reopening of the ipsi-nostril (Fig. 6A, open triangle), the cell rapidly lost its heightened responsiveness to contra-OE stimulation. After subsequent, repeated obstruction of the ipsinostril (610 s after the first obstruction, filled triangle), the cell again responded strongly to contra-OE stimulation, $\sim 160 \mathrm{~s}$ later.

A similar analysis of 27 AON neurons which responded strongly to ipsi-nasal input of an odorant category but more weakly or not at all to contra-nasal input of the same category revealed that 33\% (9 neurons) showed markedly enhanced responsiveness to contra-nasal input several minutes after ipsi-nostril obstruction.

\footnotetext{
$\leftarrow$

category component. Responses of cell \#11 to Ester category are exceptional (one case among 130 different responses listed here) in that the responses are contra-nasal selective. The odorant category marks without small circled numbers indicate that we did not determine the response to individual component odorants of the category. Filled circles, significant response; open circles, no response. $C$, The $x$-axis shows the number of odorant category matched $(0-10)$ responses of individual binasal neurons to ipsi-nasal and contra-nasal stimulation, when each of the 10 odorant categories was introduced to the ipsi- and contra-nostrils. Blue columns show the percentage of AON neurons ( $n=13$ cells) with different numbers of odorant category matches. The averaged number of odorant category matches was $\sim 8.2$. The gray bars indicate a hypothetical histogram of binominal distribution of neurons with different numbers of odorant category matches, under the assumption of random matching.
} 
However, the remaining $18 \mathrm{AON}$ neurons did not show enhanced responses to contra-nasal input, even several minutes after ipsi-nostril closure. These results indicate that deprivation of ipsi-nasal inputs causes a marked enhancement of contranasal inputs in a subset of AON neurons.

To exclude the possibility that repetitive odor stimulation of the contra-OE causes the marked enhancement of the contra-nasal inputs to the ipsi-AON neurons, we recorded $26 \mathrm{AON}$ neurons that responded to ipsi-nasal odor stimulation and repetitively stimulated the contra-OE without the ipsi-nasal obstruction. In all of the neurons, the repetitive odor stimulation for $5 \mathrm{~min}$ (at the rate of once per $25 \mathrm{~s}$ ) did not cause the heightened responsiveness to the contra-nasal odor stimulation. Furthermore, we observed in 3 neurons (among 15 neurons examined) that the subsequent ipsi-nasal obstruction caused a marked enhancement of the responses to the contra-OE stimulation with odorants. These results indicate that the ipsi-nasal obstruction but not the repetitive contra-OE stimulation causes the marked enhancement of the contra-nasal inputs in a subset of AON neurons (Fisher's exact probability test, probability of recording neurons that shows increased responses to the contra-nasal odor stimulation; 9 of 27 cells examined with ipsi-nasal obstruction, 0 of 26 cells examined without ipsi-nasal obstruction, $p<0.01$ ).

In the absence of odor stimulation, $\sim 27 \%$ (119 of 440 cells recorded) of AON neurons showed respiration phase-locked spike discharges which were presumably induced by room air odorants or periodic mechanical stimulation of the olfactory sensory neurons resulting from nasal airflow (Grosmaitre et al., 2007). These respiration-related discharges occurred at the late phase of inspiration or the initial phase of expiration (Fig. $6 B)$. The effect of ipsi-nasal obstruction was examined in $63 \mathrm{AON}$ neurons that showed room air-induced respiration phase-locked discharges. In all of these AON neurons, ipsinasal obstruction initially completely suppressed respiration phase-locked spike discharges, which suggests that respiration phase-locked inputs from the contra-OE were absent or below the threshold for inducing spike responses, just after ipsi-nasal obstruction. However, 22-292 s (138 \pm 25 s, mean $\pm \mathrm{SD}, n=17$ ) after nostril obstruction, $\sim 27 \%$ (17 of the 63 cells) began to show robust respiration phase-locked spike discharges with longer delays between inspiration and response (Figs. $6 B, 7 B$; supplemental Table 2, available at www.jneurosci.org as supplemental material). Activity exhibiting longer delays suggests that the resumed respiration phase-locked activity was induced by the remaining inputs from the contra-OE.

B
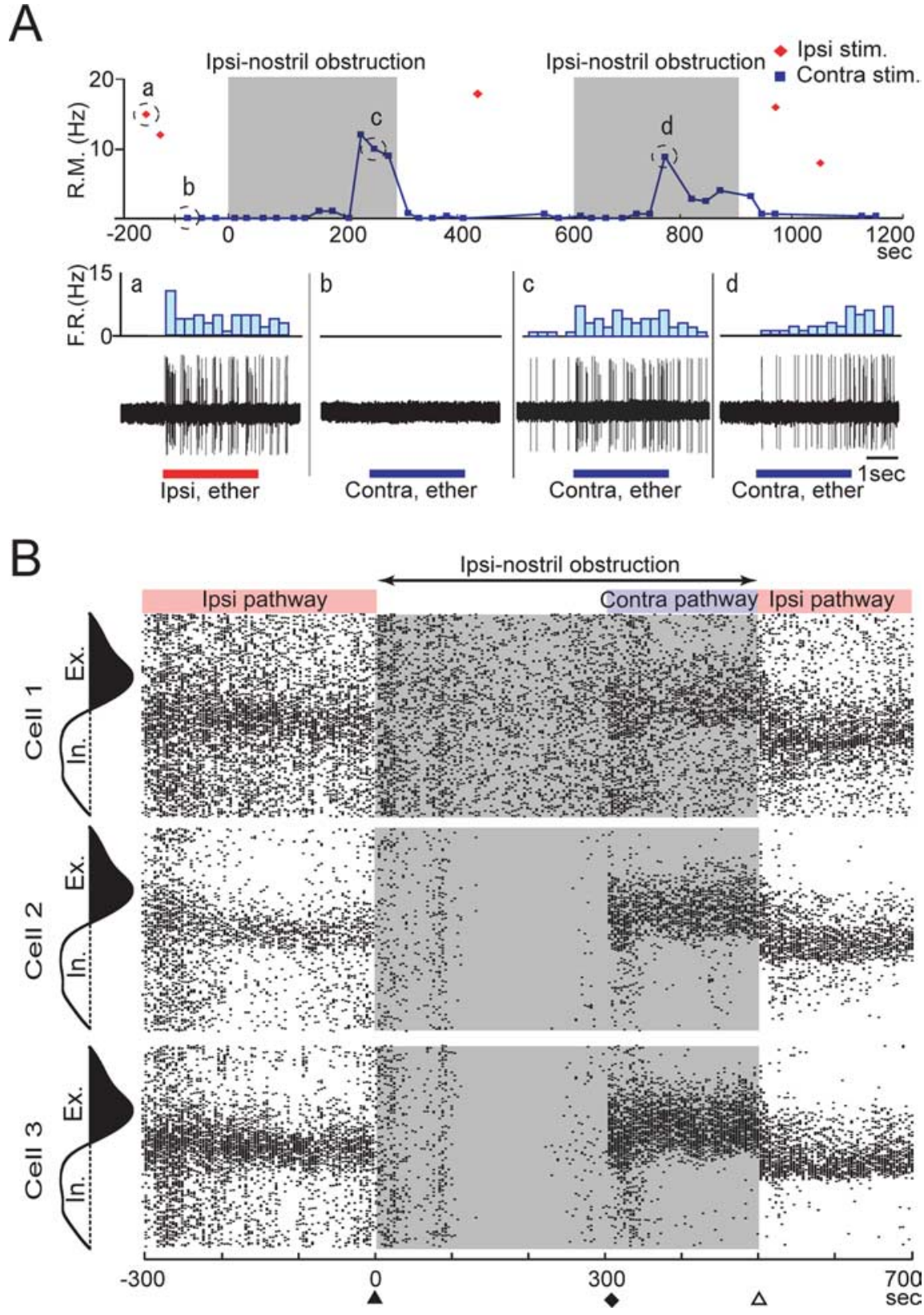

Figure 6. Ipsi-nostril obstruction rapidly enhances individual $A O N$ neuron responsiveness to contra-nasal inputs. $\boldsymbol{A}$, Time course of the response magnitude (R.M.) of an AON neuron to ipsi-OE (red diamond) or contra-OE (blue square) odorant stimulation (upper trace). Shown are firing rates (F.R.) (1 bin $=0.3 \mathrm{~s}$, middle) and spike activity (bottom) at times a, b, c, and d. This neuron responded only to ipsi-OE stimulation before ipsi-nostril obstruction ( $a$ and $b$ ), but a few minutes after ipsi-nostril obstruction ( $c$ and d) it started responding strongly to contra-OE stimulation. Filled triangle, the start of ipsi-nostril obstruction; open triangle, the time of reopening the ipsi-nostril. $\boldsymbol{B}$, Nasal obstruction enhances responsiveness to contra-nasal inputs of AON neurons. Each row (cells 1-3) indicates a raster representation of the spike discharges of an individual AON neuron during inhalation of room air, aligned with the respiratory phase. Spike responses to room air inhalation during one respiration cycle are shown on the $y$-axis (from the start of inspiration (In.) to the end of expiration (Ex.)). Responses to successive inhalations are arranged on the $x$-axis. After ipsi-nostril obstruction (at time 0 , filled triangle), the respiration phase-locked discharges of all three neurons (which peaked at the inspiration - expiration transition) disappeared, but then reappeared $\sim 300$ slater (filled diamond) with a peak at the expiration phase. Open triangle, the time of reopening the ipsi-nostril.

To examine this hypothesis, we studied the effect of ipsi-nasal obstruction on the respiration phase-locked activity of AON neurons in rats with $\mathrm{AC}$ transection ( $n=3$ rats). In $18 \mathrm{AON}$ neurons which showed respiration phase-locked spike discharges, ipsinasal obstruction completely abolished the respiration phaselocked spike discharges throughout the $5 \mathrm{~min}$ period of nostril obstruction. The absence of resumed respiration phase-locked 
A
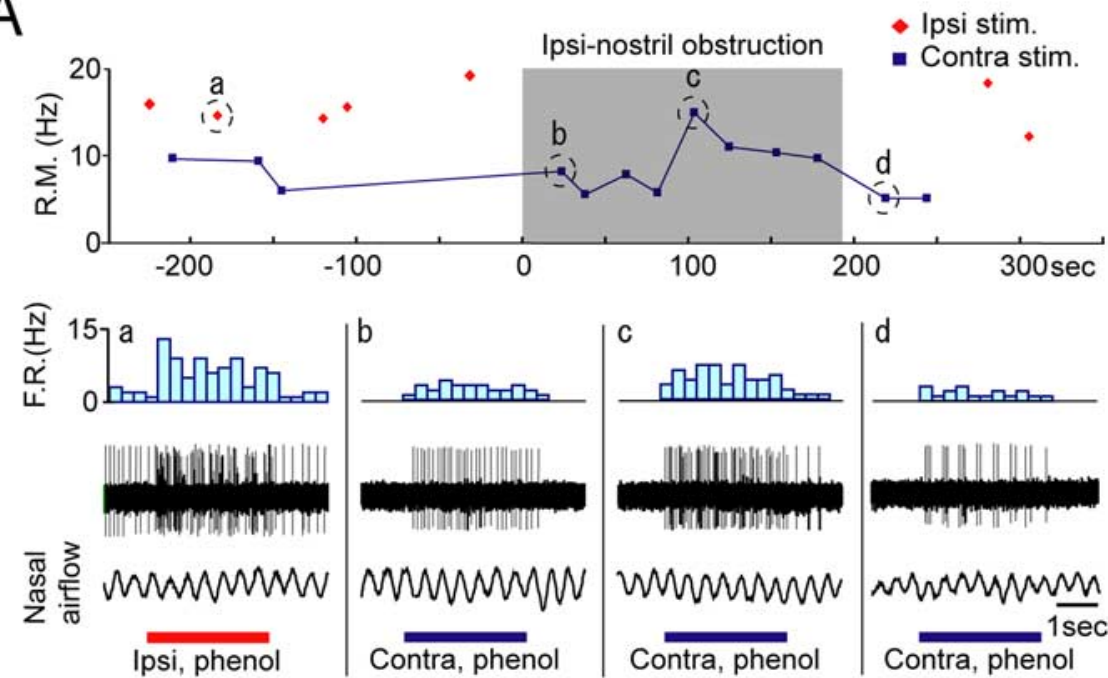

B
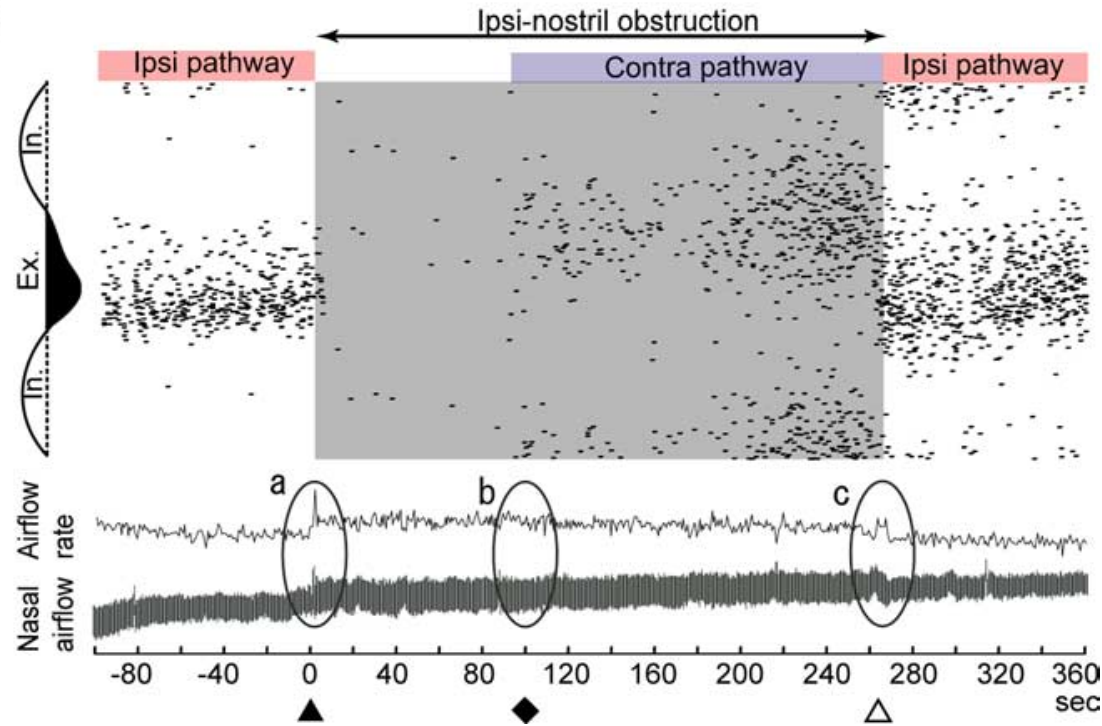

Figure 7. Enhancement of contra-nasal input occurs independently of the increase in contra-nasal airflow. $\boldsymbol{A}$, Time course of the magnitude of responses (M.R.) to ipsi-OE (red diamond) or contra- $\mathrm{OE}$ (blue square) stimulation (upper trace). Spike discharges and their histograms ( 1 bin $=0.3 \mathrm{~s}$ ) showing responses to odor stimulation at time points a, b, c, and d are shown in the middle trace. The bottom trace in each column indicates nasal airflow at the contra-nostril. The cell shown responded strongly to ipsi-0E stimulation with the phenol category $(\boldsymbol{a})$ and relatively weakly to contra-0E stimulation with the phenol category $(\boldsymbol{b})$. At $\sim 100 \mathrm{~s}$ after ipsi-nostril obstruction, the cell responded to contra-OE stimulation (c) much more strongly. No systematic change in airflow was observed at this time point. $\boldsymbol{B}$, Simultaneous recordings from an AON neuron of spike discharges (upper), airflow rate (middle), and nasal airflow at the contra-nostril (lower). Upper row indicates raster representation of the spike discharges of an AON neuron aligned with the respiratory phase [from the start of inspiration (In.) to the end of expiration (Ex.)]. In the absence of odor stimulation, the cell showed spike discharges that were phase-locked to the inhalation of the room air. After ipsi-nostril obstruction (at time 0 , filled triangle), the nasal airflow clearly increased ( $\boldsymbol{a}$ ) and then remained relatively stable, even during the period when the respiration phase-locked discharges reappeared $\sim 100$ s later $(\boldsymbol{b}$, filled diamond). Open triangle indicates the time when obstruction was removed (c).

activity in these rats indicates that the resumed respiration phaselocked inputs observed in rats with intact $\mathrm{AC}$ are mediated via the AC (Fisher's exact probability test, probability of recording neurons that show resumed phase-locked firing after ipsi-nasal obstruction; 17 of 63 cells examined ( $\sim 27 \%)$ in the AC intact group, and 0 cells out of 18 cells $(0 \%)$ in the AC transected group, $p<0.01)$

The increased response of the AON neurons to contra-nasal inputs after ipsi-nasal blockage may be the result of the plastic mechanism of the neuronal pathway from the contra-OE via
contra-OB, contra-AON/OC to the ipsiAON. However, there remains the possibility that the increased response of AON neurons is simply caused by increased air flow in the contra-nasal passage because of the blocking of the ipsi-nasal passage. To examine the latter possibility, we recorded responses of AON neurons before and after ipsilateral nostril closure, while simultaneously monitoring the air flow in the contra-nostril by using a thermocouple. We found that the airflow rate in the contra-nostril increased at the onset of ipsi-nasal obstruction; however, the airflow was unchanged at the time the neurons suddenly developed enhanced responsiveness to contra-OE stimulation (Fig. 7). Although the lack of temporal correlation between the onset of the increased airflow and the onset of the increased response does not necessarily rule out the possibility of the causal relationship, the results indicate that the increased responses cannot be explained solely by the increased airflow. These observations suggest that the central olfactory system contains a neuronal mechanism that switches nasal inputs to the contra-OE when ipsi-nasal inputs are blocked.

\section{Discussion}

These results showed that in binasal E-E (ipsi-excitation, contra-excitation) type AON neurons, odorant category selectivity of contra-nasal inputs closely resembled that of the ipsi-nasal inputs. Individual binasal neurons thus receive similar odor information from the ipsi-nasal pathway and the contra-nasal pathway (compare Fig. 1). These binasal AON neurons may be involved in the neuronal mechanisms for the transfer of ipsi-nasal olfactory information to the contralateral OC via the AC (Kucharski and Hall, 1987).

Convergence of similar sensory information on individual neocortical neurons from right and left sensory organs has been reported in visual and auditory systems (Hubel and Wiesel, 1962; Middlebrooks et al., 1980). Detection by these sensory cortices of subtle signal differences between bilateral sensory organs facilitates computation of visual depth and localization of a sound source, respectively (Barlow et al., 1967; Knudsen and Konishi, 1979). The olfactory system also has the ability to localize the odor source (Rajan et al., 2006; Von Bekesy, 1964; Porter et al., 2005). Thus, one possible function of the convergence of nearly equivalent olfactory signals from bilateral OEs is to detect the subtle difference of signals from the two OEs, which may be the role played by subpopulations of E-E (ipsi-excitation, contra-excitation) type and E-I (ipsi-excitation, contra-suppression) type AON neurons. Another important function of equivalent convergence is to maintain a reserve of 
sensory input, so that in the event that one sensory organ is temporarily not functional, the brain can monitor the external world through the remaining intact sensory organ.

Our results showed that a majority of AON neurons were either binasal E-E (ipsi-excitation, contra-excitation) type or ipsi-nasal type, and that in the E-E (ipsi-excitation, contraexcitation) type AON neurons, ipsi-nasal inputs exceeded contra-nasal inputs in magnitude. Thus, the activity of AON neurons is ordinarily dominated by ipsi-nasal inputs. In addition, we showed that ipsi-nostril obstruction rapidly enhanced, within several minutes, the contra-nasal input of a subset of AON neurons in a reversible manner. These results indicate a novel form of binostril competition in the adult OC.

Congestion of nasal mucosa is thought to be a necessary step for mucous clearance (Eccles, 2000b); however, the process inevitably alters nasal airflow and the concomitant activity of the left and right OEs. To cope with the reciprocal change in OE activity, the central olfactory system may have evolved a homeostatic plasticity mechanism of neural circuits for rapidly adjusting the magnitude of contra-nasal inputs based on the magnitude of ipsi-nasal inputs, such that at least a subset of AON neurons can continue to respond to external odors. As a first step to understand the plasticity of the central olfactory system, we examined the change in the contra-nasal input after a complete obstruction of the ipsi-nasal passage. Because the natural nasal obstruction is almost always a partial obstruction, further experiments are necessary to examine the responses to contra-nasal stimulation after partial and graded obstructions of the ipsi-nasal passage.

A global homeostatic mechanism that provides stability to neuronal networks has been reported to work in the juvenile mouse visual cortex during ocular dominance plasticity (Mrsic-Flogel et al., 2007). Monocular deprivation results in decreased deprived-eye inputs, increased non-deprived eye inputs in neurons that receive binocular inputs, and increased deprived-eye responses in neurons devoid of non-deprived eye inputs (Mrsic-Flogel et al., 2007), indicating that the response adjustment effectively preserves the net visual drive for individual neurons. We speculate that a similar homeostatic plastic mechanism may work in the adult olfactory system to preserve the net olfactory drive for each AON neuron. A previous study showed that unilateral naris obstruction for $>30 \mathrm{~d}$ enhances the intracortical association fiber input to the olfactory cortex of the adult rat (Best and Wilson, 2003). Unilateral naris obstruction for $5 \mathrm{~d}$ causes downregulation of the NMDA receptor NR2B subunit and phospho-CREB, specifically in layer IIb pyramidal cells of the mouse OC (Kim et al., 2006). These results raise the possibility that similar changes may occur in the AON during rapid compensatory switching of binasal inputs. Further work is necessary to elucidate the cellular, synaptic, and molecular mechanisms of AON rapid plasticity.

It remains to be determined whether ipsi-nasal obstruction causes any change in the responses of mitral cells in the contra-OB to the contra-nasal odor stimulation. If there are changes in the mitral cell responses, future studies should address the question to what extent the plasticity of $\mathrm{AON}$ neurons is caused by the altered outputs of mitral cells in the ipsi- and contra-OBs.

A characteristic feature of mono-nasal deprivation-induced nasal dominance plasticity is its extreme rapidness; the increase in responsiveness to contra-nasal inputs occurs within several minutes after the onset of ipsi-nostril closure. This is in striking contrast to the visual and auditory systems which require longterm deprivation of unilateral sensory input to induce the plastic change of central neuron responses to input from the nondeprived sensory organ. In the adult mouse visual cortex, $>2 \mathrm{~d}$ of monocular deprivation is required to induce homeostatic plastic changes (Goel and Lee, 2007). In the inferior colliculus of the adult ferret auditory system, $1 \mathrm{~d}$ removal of input from the dominant, excitatory contralateral ear is needed to induce enhanced excitation from the ipsilateral ear (McAlpine et al., 1997). The rapid nature of the mono-nasal deprivation-induced nasal dominance plasticity in the $\mathrm{AON}$ of adult rats provides a useful model system with greater experimental tractability for analyzing the cellular and molecular mechanisms of experience-dependent cortical plasticity in the adult brain.

Rapid switching of binasal inputs to a subset of AON neurons may enable both right and left olfactory cortices to continuously monitor the external odor world despite unilateral and reciprocal obstruction of the nasal airflow. This objective is further facilitated by the fact that individual neurons of this subset obtain similar olfactory information from the ipsi- and contra-OEs.

\section{References}

Barlow HB, Blakemore C, Pettigrew JD (1967) The neural mechanism of binocular depth discrimination. J Physiol 193:327-342.

Best AR, Wilson DA (2003) A postnatal sensitive period for plasticity of cortical afferents but not cortical association fibers in rat piriform cortex. Brain Res 961:81-87.

Bojsen-Moller F, Fahrenkrug J (1971) Nasal swell-bodies and cyclic changes in the air passage of the rat and rabbit nose. J Anat 110:25-37.

Brunjes PC, Illig KR, Meyer EA (2005) A field guide to the anterior olfactory nucleus (cortex). Brain Res Rev 50:305-335.

Eccles RB (2000a) Nasal airflow in health and disease. Acta Otolaryngol 120:580-595.

Eccles RB (2000b) The nasal cycle in respiratory defence. Acta Otorhinolaryngol Belg 54:281-286.

Goel A, Lee HK (2007) Persistence of experience-induced homeostatic synaptic plasticity through adulthood in superficial layers of mouse visual cortex. J Neurosci 27:6692-6700.

Grosmaitre X, Santarelli LC, Tan J, Luo M, Ma M (2007) Dual functions of mammalian olfactory sensory neurons as odor detectors and mechanical sensors. Nat Neurosci 10:348-354.

Haberly LB, Price JL (1978) Association and commissural fiber systems of the olfactory cortex of the rat. II Systems originating in the olfactory peduncle. J Comp Neurol 178:781-808.

Hensch TK (2005) Critical period plasticity in local cortical circuits. Nat Rev Neurosci 6:877-888.

Hubel DH, Wiesel TN (1962) Receptive fields, binocular interaction and functional architecture in the cat's visual cortex. J Physiol 160:106-154.

Kim HH, Puche AC, Margolis FL (2006) Odorant deprivation reversibly modulates transsynaptic changes in the NR2B-mediated CREB pathway in mouse piriform cortex. J Neurosci 26:9548-9559.

Knudsen EI, Konishi M (1979) Mechanisms of sound localization in the barn owl. J Comp Physiol 133:13-21.

Kucharski D, Hall WG (1987) New routes to early memories. Science 238:786-788.

Lei H, Mooney R, Katz LC (2006) Synaptic integration of olfactory information in mouse anterior olfactory nucleus. J Neurosci 26:12023-12032.

McAlpine D, Martin RL, Mossop JE, Moore DR (1997) Response properties of neurons in the inferior colliculus of the monaurally deafened ferret to acoustic stimulation of the intact ear. J Neurophysiol 78:767-779.

Middlebrooks JC, Dykes RW, Merzenich MM (1980) Binaural responsespecific bands in primary auditory cortex (AI) of the cat: topographical organization orthogonal to isofrequency contours. Brain Res 181:31-48.

Mori K, Nagao H, Yoshihara Y (1999) The olfactory bulb: coding and processing of odor molecule information. Science 22:711-715.

Mrsic-Flogel TD, Hofer SB, Ohki K, Reid RC, Bonhoeffer T, Hübener M (2007) Homeostatic regulation of eye-specific responses in visual cortex during ocular dominance plasticity. Neuron 54:961-972.

Murakami M, Kashiwadani H, Kirino Y, Mori K (2005) State-dependent sensory gating in olfactory cortex. Neuron 46:285-296. 
Nagayama S, Takahashi YK, Yoshihara Y, Mori K (2004) Mitral and tufted cells differ in the decoding manner of odor maps in the rat olfactory bulb. J Neurophysiol 91:2532-2540.

Neville KR, Haberly LB (2004) Olfactory cortex In: The synaptic organization of the brain (Shepherd GM, ed), pp 415-454. New York: Oxford UP.

Porter J, Anand T, Johnson B, Khan RM, Sobel N (2005) Brain mechanisms for extracting spatial information from smell. Neuron 18:581-592.

Rajan R, Clement JP, Bhalla US (2006) Rats smell in stereo. Science 311:666-670.

Sawtell NB, Frenkel MY, Philpot BD, Nakazawa K, Tonegawa S, Bear MF (2003) NMDA receptor-dependent ocular dominance plasticity in adult visual cortex. Neuron 38:977-985.
Schoenfeld TA, Macrides F (1984) Topographic organization of connections between the main olfactory bulb and pars externa of the anterior olfactory nucleus in the hamster. J Comp Neurol 227:121-135.

Scott JW, McBride RL, Schneider SP (1980) The organization of projections from the olfactory bulb to the piriform cortex and olfactory tubercle in the rat. J Comp Neurol 194:519-534.

Von Bekesy G (1964) Olfactory analogue to directional hearing. J Appl Physiol 19:369-373.

Wilson DA (1997) Binaral interactions in the rat piriform cortex. J Neurophysiol 78:160-169.

Yoshida I, Mori K (2007) Odorant category profile selectivity of olfactory cortex neurons. J Neurosci 27:9105-9114. 